\title{
Application Research of CBCT in Orthodontics
}

\author{
Hongyu $\mathrm{Xu}^{*}$, Yuanjun Wang \\ School of Medical Instrument and Food Engineering, \\ University of Shanghai for Science and Technology \\ Shanghai, China \\ E-mail: xhongyu69@163.com
}

\begin{abstract}
CBCT (Cone Beam Computer Tomography) has been widely used in various fields of oral cavity because of its high resolution and low radiation. Through the comparis on between CBCT and traditional spiral CT, it showed the advantages of CBCT in orthodontics field and exampled how to use CBCT for the diagnos is and examination of oral dise ases. It thoroughly explained how CBCT technology can be applied to orthodontics, such as $3 \mathrm{D}$ cephalometry, location of impacted teeth and supernumerary teeth, orthodontic expansion, 3D reconstruction and analys is of bone mass. It explains that CBCT technology is a leap of oral 3D imaging technology. The reconstruction method can match the oral maxillofacial anatomy characteristics, and can provide 3D images of high quality for clinicians. It is useful to understand the disease directly, accurately, and totally. It is great significance to improve the reliability, the level of treatment and the prognostic evaluation of the clinical diagnosis.
\end{abstract}

Keywords-CBCT; Orthodontics; 3D cephalometry.

The most common imaging data of Orthodontics are traditional 2D images, such as panorama and X-ray cranial lateral films. For years, the images between the teeth and the jaw are mainly used to evaluate the maxillofacial malformation. The traditional $\mathrm{X}$ - ray dental film and panoramic radiograph can only observe the craniofacial bone, the dentition and the root tip in 2D space, but it can't display the facial soft tissue clearly. So the orthodontic reference anatomy is easy to distort, and the patient's facial space is not enough clear ${ }^{[1]}$. In recent years, special dental CT(dental CT, CBCT) has been introduced gradually, and has been used in oral orthodontics. Especially, the CBCT has been used in oral craniofacial because of its high accuracy, low radiation and 3D imaging ${ }^{[2]}$.

\section{ADVANTAgES OF CBCT IN ORTHODONTICS}

From the imaging structure, CBCT uses a 3D conical beam X-ray scan instead of the 2D fan beam scanning of the body layer CT. After the projection data is reconstructed, the 3D image can be obtained directly. Accordingly, it uses a 2D surface detector to replace the linear detector of the body layer CT. Obviously, the use of conical beam X-ray scan can significantly improve the utilization of X-ray. After 360 degrees of rotation, all the original data for reconstruction obtained. Collecting projection data with a surface detector can accelerate the speed of data acquisition. In addition, compared with traditional CT, CBCT scan range is flexible, which can scan not only specific diagnosis area but also all craniofacial part. The images formed by CBCT have higher precision, and can be measured as real size as the shot. The scanning time is shorter and the radiation dose is smaller.

TABLE I. Comparison of Main Performances between CBCT and

\begin{tabular}{|c|c|c|}
\multicolumn{2}{c}{} & Traditional CT \\
\hline Imaging range & small & Traditional CT \\
\hline Spatial resolution & high & big \\
\hline Scanning speed & fast & relatively low \\
\hline Radiation dose & low & high \\
\hline Density resolution & low & high \\
\hline Pixel uniformity & isotropy & $\begin{array}{c}\text { anisotropy } \\
\text { (under 16 row) }\end{array}$ \\
\hline Size and weight & small and light & large and heavy \\
\hline Price & cheap & expensive \\
\hline
\end{tabular}

With the development of orthodontics, the application of 3D $\mathrm{CT}$ is becoming more and more common. Some developed areas have taken CBCT as a regular inspection method for oral and maxillofacial imaging due to the advantages of higher precision, shorter scanning time, smaller radiation and lower price.

\section{ApPLICATION OF CBCT IN ORTHODONTICS}

\section{A. 3D Cephalometrics of Orthodontics}

In the clinical diagnosis of orthodontics, cephalometrics is an important analysis method, which provides the basis for the treatment design, the evaluation of the curative effect and the related research work. The current common 3D cephalometric methods include X-ray skull stereoscopic photography, 3D CT and CBCT measurement. CBCT measurement can extract all kinds of tissues from the original data of patients. The results are accurate and the operation is easy. There is no unified standard for 3D cephalometric analysis. When using CBCT for cephalometric analysis, the software uses the data obtained from CBCT to generate the head anterioposterior and lateral film, then output to the cephalometric analysis software for digital measurement. The volume data obtained by one CBCT scan can be well adapted to the pre orthodontic clinical examination after the $3 \mathrm{D}$ reconstruction processing. To some extent, it may replace the panoramic radiograph and traditional cephalometric films and become a routine examination before orthodontics.

\section{B. Location of Impacted Teeth and Supernumerary Teeth}

At present, the clinical diagnosis of impacted teeth is usually realized by routine oral examinations, $X$ - ray tooth films and oral maxillofacial panoramic films. However, due to the influence of the anatomical structure of bones and the projection angle, the image distortion and the structure overlap often exist in the $\mathrm{X}$ - ray tooth films and oral maxillofacial panoramic films. 
It is difficult to find the root of infection accurately. CBCT can get a complete image by a single scan. There is no gap in data acquisition and 1:1 ratio imaging, so the image is more accurate. CBCT scan time is much shorter than traditional multi-slice spiral CT, usually only about 20s. This is not only more convenient for patients, but also less radiation amount of patients due to the decrease of scanning time. Its radiation dose is only the $1 / 30 \sim 1 / 100$ of traditional multi-slice spiral CT, which will not affect the health of the patients. CBCT can create $3 \mathrm{D}$ reconstruction images after selecting the rebuilt range by embedded software, and carry out an arbitrary angle rotation observation. It can accurately display the position and depth of supernumerary teeth in the jaw bones, and it has obvious advantages in reflecting the shape, size, quantity, direction, position in the lip and palate, and the relationship with the nose and the adjacent teeth. Through the 3D reconstruction technique and the adjustment of window width and position, the images can remove some bone tissue and leave a high density tooth image. It avoids the overlapping of surrounding tissue structure affects the location of the supernumerary teeth.

\section{Application of CBCT in Orthodontic Expansion}

The application of CBCT in orthodontic expansion is used to obtain the 3D data model of the craniofacial face of clinical patients, and establish a 3D coordinate system by the inherent anatomical marks of the cranium. Then the correction changes are compared directly under the same measuring coordinate system, which can observe the changes of the position and angle between the mark points of jaw teeth before and after the rapid expansion. The main reactions after rapid expansion are bone expansion, alveolar bone expansion and tooth inclination. CBCT can measure the opening of palatine suture, the widening of the dental arch and the movement of the root crown of posterior teeth under expansions, so it can quantitatively evaluate the tissue responses in the expansion, which provides a basis for studying the biomechanical mechanism of rapid expansions. From the clinical applications of Garrett $\mathrm{BJ}^{[3]}$ and Rungcharassaeng $\mathrm{K}^{[4]}$ in orthodontic expansion, they prove that CBCT is a safe and reliable method to study the changes of the related tissue after rapid expansion. It can better analyze the widening of the bone seams, tooth tilt and the remodeling of the cheek of the alveolar bone after the expansion. With the help of the DICOM image data of CBCT, F.A. Zabir ${ }^{[5]}$ built the maxillary expansion model by using Mimics software, which provides an effective tool for further studying the mechanism of expansion and the distribution of stress.

\section{Evaluation of Bone Mass}

In orthodontics, the most popular clinical application is bone mass analysis using CBCT to evaluate preoperative dental implant surgery. Next, it will example how CBCT judges the patient's condition and determines the plan in anterior teeth implant surgery.

\section{1) urate Preoperative Assessment}

After the loss of anterior teeth, the absorption of alveolar bone is very complicated by the factors such as the degree of lesion, time and the absence of teeth. It is difficult to know the true status of the alveolar bone in the anterior teeth only through oral examination or traditional 2D image. Sometimes, it finds that patients who can do implantation at clinical examination don't have enough bone volume of the alveolar bone through CBCT further examinations and are not suitable for operation. Therefore, adequate dental examination and accurate preoperative evaluation should be made before anterior dental implant surgery, and the best way will be determined.

\section{2) Correct Judgement for Alveolar Bone}

The lack of bone mass in the anterior teeth is often found. The most common problem in young and middle-aged patients is the lack of alveolar bone thickness, or the lack of bone mass caused by obvious deformation, which affects the implantation path. In the same patient's anterior teeth, the shape of alveolar bones in the different location is not consistent. Some alveolar bones are irregular, and their heights will also be inadequate at tooth lack areas in the long time. CBCT examination can get bone height, width and bone density, which is helpful for clinicians to determine implant angle and ensure implant plan.

\section{3) Selecting Corresponding Surgical Methods}

When the insufficiency of bone mass is found before implanting operations, CBCT examination can know the loss of bone and the defect site, which helps clinicians select the different surgical methods to implant bone in implanting site, so that obtain enough bone mass for the implant growth. If the alveolar bone mass is obviously insufficient by the CBCT examination, the surgeon can implement the implanting bone operation primarily. After implanted bone and alveolar bone grown and healed, the second stage implanting operation was performed.

\section{E. Dental Root Resorption Diagnosis}

CBCT can accurately reflect root resorption site, lesion area and its relationship with surrounding tissues in $3 \mathrm{D}$ space. As the detection rate of root resorption is increasing, CBCT becomes the most effective diagnostic tool in clinical examination, and its high resolution provides strong evidence for preventive tooth extraction. In addition, the key to determine the effect of root resorption is to find and predict the occurrence of root resorption as soon as possible. The accuracy of CBCT also brings great convenience to the evaluation of the sick teeth. For example, to diagnose and treat the external tooth root resorption caused by impacted teeth, Li et al ${ }^{[6]}$ scanned 19 teeth and reconstructed them. The generated images showed that CBCT could display the location and degree of the root resorption directly and accurately, and provide reliable information for the diagnosis and treatment of the external tooth root absorption caused by impacted teeth. Liedke GS et al ${ }^{[7]}$ analyzed the root absorption reliability of 59 teeth using CBCT with different resolution, and found that the $0.3 \mathrm{~mm}$ pixel resolution is the best setting condition for the diagnosis of root absorption. It confirmed that CBCT examination can provide comprehensive and reliable image information with high resolution. Chen ${ }^{[8]}$ used CBCT to analyze the $3 \mathrm{D}$ effects of lower resist compression of micro implant nails than long teeth and the feasibility of the root absorption. The result confirmed that CBCT can accurately obtain the measured data of each tooth and each tooth tip. The above research shows that CBCT technology can accurately detect the incidence of root resorption in clinical diagnosis with its high resolution.

\section{F. Measurements of Temporomandibular Joint}

Through correct placement, scanning and image reconstruction, CBCT can obtain the image of the axial, sagittal, 
coronal position of the temporomandibular joint, the oblique position of parallel and perpendicular to the long axis of the condyle, and double condyle surfaces. By adjusting the contrast and the grayscale, clinicians can make a comprehensive and accurate diagnosis for the morphology and location of the bony structures of the temporomandibular joint ${ }^{[9]}$. Although the imaging of spiral CT is clearer than that of CBCT, CBCT has the advantages in low cost, simple operation and rapid imaging, which makes it have a wider development prospect in Orthodontics. After 3D reconstruction of the temporomandibular joint, CBCT can obtain the images of different parts and layers through different tangent surfaces, and can clearly display the morphological structure of the condyle, joint concave, joint nodule and joint space. It also can measure the condylar horizontal angle, the condyle axis area, the distance of the two poles and the radius distance between unilateral condyle and sagittal line. Through 3D reconstruction and image registration, CBCT can directly evaluate the shape and position changes of condyle in treatment.

\section{G. 3D Reconstruction}

CBCT can be used to evaluate the bone density, bone mass and the position of the mandibular canal, and to maximize the use of data measurement, which avoids the interference caused by the lack of bone mass, and reduces the failure rate of the operation. For example, $\mathrm{Wu}$ et $\mathrm{al}^{[10]}$ used the Kodak digital CBCT and Kodak Dental Image Software to make a study model for 75 patients of oral diseases filmed by CBCT. He analyzed the indications of dental implant surgery based on the data obtained from the images, then made perfect operation plans, and designed the diameter, length and implantation angle of implants according to the direct simulation positioning in the image. Through data analysis, the operation time of all the patients is obviously shortened, which relieves the pain of the patients. The short-term successful rate of operations was up to $100 \%$ after 3 months by CBCT observation.

\section{CONCLUSION}

CBCT technology is a leap in the development of oral 3D imaging technology. It can provide clinicians with high quality 3D images, which helps clinicians to grasp the status of the disease intuitively, accurately and integrally, and improves the reliability, treatment level and prognosis evaluation of clinical diagnosis. However, CBCT also has some shortcomings. Its gray resolution is lower, the range of projection is smaller, and the reduction of soft tissue is not as good as MRI. At present, the imaging of vascular diseases in the maxillofacial region has not been developed. There is still a certain missed diagnosis rate and misdiagnosis rate in the diagnosis of mild root resorption by CBCT. In the future, how to improve the diagnostic accuracy of CBCT without increasing the dose of radiation is a hot topic in CBCT research. Moreover, with the continuous development of system configuration and software, CBCT will hopefully become the most ideal imaging equipment for orthodontics.

\section{REFERENCES}

[1] Wei Haidong and Xu Bo. "The development of CT imaging in orthodontic orthodontics”[J]. China Modern Doctor. July 2010. Vol 48.No 20. 1673-9701.(In Chinese).

[2] Hu Haifeng, Zang Li, Chen Zhiqiang, Li Liang, Wu Hong xin and Li Yingcao. “Application of cone beam computed tomography technology in oral clinical practice”[J]. Computerized Tomography Theory and Applications, Sep 2009. Vol 18, No 3. 30-37. (In Chinese) .

[3] Garrett BJ, Caruso JM, Rungcharassaeng K, Farrage JR, Kim JS and Taylor GD. "Skeletal effects to the maxilla after rapid maxillary expansion assessed with cone-beam computed tomography” [J]. Am J Orthod Dentofacial Orthop. July 2008. Vol 134,No 1: 8-9.

[4] Rungcharassaeng K, Caruso JM, Kan JY, Kim J and Taylor G. "Factors affecting buccal bone changes of maxillary posterior teeth after rapid maxillary expansion” [J]. Am J Orthod Dentofacial Orthop 2007. Vol 132,No 4:1-8.

[5] F. A. Zabir, A. S. Abdullah, N. A. Abu Osman, Z. Radzi, N. A. Yahya and N. H. Abu Kasim. "Acquiring anatomical representation of human maxilla for rapid maxillary expansion”. 4th Kuala Lumpur International Conference on Biomedical Engineering 2008 BIOMED 2008 25-28 June 2008 Kuala Lumpur, Malaysia.

[6] Li Zhijin, Guo Jiaping, Shi Yongmei, Dong Qingshan, Wang Xiang, Xiong Caihua and Gui Xiao. "Application of conical beam CT in the diagnosis and treatment of external tooth root resorption caused by impacted teeth”[J]. West China Journal of Stomatology .Dec 2013.Vol 31,No 6:588-591. (In Chinese).

[7] Liedke GS, da Silveira HE, da Silveira HL, Dutra V and de Figueiredo JA. "Influence of voxel size in the diagnostic ability of cone beam tomography to evaluate simu lated external root resorption” [J]. J Endod. Feb 2009. Vol 35.No 2:233-235.

[8] Chen Fei. "CBCT analysis of low overgrown teeth with micro implant nails”. Zhejiang University Doctoral. 2010. (In Chinese).

[9] Cao Junkai, Wang Zhaowu, Liu Hongchen, Hu Min and Jiang Hua. "The measurement technique of skeletal structure of temporo mandibular joint with dental volumetric computerized tomography”[J]. Chinese Journal of Prosthodontics. Oct, 2008. Vol,9.No,4:291-294. (In Chinese).

[10] Wu Haoyang, Huo BeiBei and Wu Dong. "Clinical application of 3D CBCT imaging system at oral implant”[J]. Chinese Journal of Oral Implantology. 2011. 16(2):125-127. (In Chinese). 